\title{
Prevention of Bloodstream Infections With Central Venous Catheters Treated With Anti- Infective Agents Depends on CATHeTer Type AND InsERTION Time: EVIDENCE From A META-ANALYsis
}

\author{
Bernhard Walder, MD; Didier Pittet, MD, MSc; Martin R. Tramèr, MD, DPhil
}

\begin{abstract}
OBJECTIVE: 'To test the evidence that the risk of infection related to central venous catheters (CVCs) is decreased by antiinfective coating or cuffing.

DESIGN: Systematic review of randomized, controlled trials comparing anti-infective with inactive (control) CVCs.

INTERVENTIONS: Average insertion times were taken as a measurement of the length of insertion. Dichotomous data were combined using a fixed effect model and expressed as odds ratio $(\mathrm{OR})$ with $95 \%$ confidence interval $\left(\mathrm{CI}_{95}\right)$.

RESULTS: Two trials on antibiotic coating ( $343 \mathrm{CVCs}$ ) had an average insertion time of 6 days; the risk of BSI decreased from $5.1 \%$ with control to $0 \%$ with anti-infective catheters. There were no trials with longer average insertion times. In three trials on silver collagen cuffs ( $422 \mathrm{CVCs}$ ), the average insertion time ranged from 5 to 8.2 days (median, 7 days); the risk of BSI was $5.6 \%$ with control and $3.2 \%$ with anti-infective catheters. In another trial on silver collagen cuffs ( $101 \mathrm{CVCs})$, the average insertion time was 38 days;
\end{abstract}

Central venous catheters (CVCs) are commonly used for hemodynamic monitoring, administration of medication, and parenteral nutrition. The most frequently reported problems are insertion complications, occlusion of the catheter, vascular thrombosis, and catheter-related infections. ${ }^{1}$ Infection may be local or systemic, including thrombophlebitis, bloodstream infection (BSI), endocarditis, and metastatic distribution (eg, osteomyelitis, endophthalmitis, or arthritis). ${ }^{2,3}$ CVC-related bacteremia is a major cause of nosocomial BSI, 4,5 with a reported incidence of approximately $5 \% .{ }^{6}$ The consequences of these infections, in terms of morbidity, mortality, and additional healthcare costs, are of major importance.?

A novel technologic approach to reduce CVC-related infection is the impregnation of catheters with antiseptic or antimicrobial agents, but clinical trials to assess the efficacy of these agents have produced inconsistent results. 8,9 Guidelines for the prevention of intravascular device-related infections published by the Centers for Disease Control the risk of BSI was $3.7 \%$ with control and $4.3 \%$ with anti-infective catheters. In five trials on chlorhexidine-silver sulfadiazine coating $(1,269 \mathrm{CVCs})$, the average insertion time ranged from 5.2 to 7.5 days (median, 6 days); the risk of BSI decreased from $4.1 \%$ with control to $1.9 \%$ with anti-infective catheters. In five additional trials on chlorhexidine-silver sulfadiazine coating $(1,544 \mathrm{CVCs})$, the average insertion time ranged from 7.8 to 20 days (median, 12 days); the risk of BSI was $4.5 \%$ with control and $4.2 \%$ with anti-infective catheters.

CONCLUSIONS: Antibiotic and chlorhexidine-silver sulfadiazine coatings are anti-infective for short (approximately 1 week) insertion times. For longer insertion times, there are no data on antibiotic coating, and there is evidence of lack of effect for chlorhexidine-silver sulfadiazine coating. For silver-impregnated collagen cuffs, there is evidence of lack of effect for both short- and long-term insertion (Infect Control Hosp Epidemiol 2002;23:748$756)$

and Prevention proposed the use of impregnated catheters for adults with an unacceptably high rate of infection, ${ }^{10}$ but no consensus has been reached about the definition of such a rate.

Recently, three studies reported important data on this subject. Two meta-analyses concluded that catheters impregnated with chlorhexidine-silver sulfadiazine decreased the incidence of catheter-related BSIs by $2.2 \%$ compared with inactive control catheters. ${ }^{11,12}$ However, a large, randomized, controlled trial showed that catheters treated with minocycline and rifampin were more efficacious in preventing BSI compared with those treated with chlorhexidine-silver sulfadiazine. ${ }^{13}$ In a few catheters with prolonged insertion times ( $>1$ week), an increased rate of BSI was observed for those treated with chlorhexidine-silver sulfadiazine compared with those treated with antibiotics. Although the numbers were small, it was suggested that these results should lead to a change in clinical practice for critical care patients. ${ }^{14}$

Dr. Walder is from the Division of Surgical Intensive Care, Dr. Tramèr is from the Division of Anaesthesiology, Department APSIC, and Prof. Pittet is from the Infection Control Program, Department of Internal Medicine, University of Geneva Hospitals, Geneva, Switzerland.

Address reprint requests to Bernhard Walder, MD, Division of Surgical Intensive Care, University of Geneva Hospitals, 24 rue Micheli-du-Crest, CH-1211 Geneva 14, Switzerland.

Dr. Tramer was supported by a PROSPER grant from the Swiss National Science Foundation (no. 3233-051939.97).

The author thank Daniel Haake from the Documentation Service of the Swiss Academy of Medical Sciences for his assistance in searching electronic databases and Rosemary Sudan for editorial assistance. 
We undertook this study of randomized, controlled trials to quantify the relative efficacy of $\mathrm{CVCs}$ treated with different anti-infective agents and to identify factors that have an impact on the efficacy of these devices.

\section{METHODS}

\section{Search Strategy}

An extensive search of the relevant literature in all languages was performed using MEDLINE (Data Star, PubMed, and KnowledgeFinder 4.19 to January 20, 2000), EMBASE (to January 12, 2000), and Cochrane Library (2000, issue I). Key words used alone or in combination included central venous catheter, infection, coated, impregnated, subcutaneous cuff, antibiotic bonding, antibioticcoated, silver-sulfadiazine, chlorhexidine, and random. Reference lists of retrieved reports, review articles, ${ }^{10}$ and meta-analyses ${ }^{11,15}$ were checked. Data from abstracts, letters, review articles, and animal studies were not considered.

\section{Inclusion Criteria, Endpoints, and Definitions}

Included studies were full reports of randomized, controlled trials of adults that were published in peerreviewed journals and that compared any antiseptic- or antimicrobial-coated or antiseptic- or antimicrobial-cuffed CVC (anti-infective) with a similar uncoated or uncuffed device (control). Relevant trials had to report on catheters that were inserted percutaneously; those in which catheters were exchanged over a guidewire were not considered.

One of two endpoints had to be reported in dichotomous form according to the definitions provided by the original authors: catheter colonization per 100 catheters inserted and BSI per 100 catheters inserted. Catheter colonization was considered as less important. It was defined as a documented growth from a proximal or distal catheter segment of either 15 or more colony-forming units (CFU) in a semiquantitative culture ${ }^{16}$ or more than $10^{3} \mathrm{CFU}$ in a quantitative culture. ${ }^{2}$ This definition was valid for the purpose of this study regardless of whether cultures were obtained from the external surface only or from both the internal and the external surfaces of the catheters. BSI was considered as the primary endpoint in this context. It was defined as the presence of the same organism isolated from a (semi) quantitative culture of a catheter segment and from the patient's blood in the absence of another source of infection. ${ }^{5}$ This subgroup included patients with or without accompanying clinical symptoms (ie, sepsis).

\section{Scoring and Data Extraction}

All authors independently read the included articles and assessed their methodologic quality using the validated Oxford scale. ${ }^{17}$ There was a pre hoc agreement to include trials with randomization according to the patients' date of birth, hospital chart number, or alternate allocation, but to exclude trials without randomization (eg, historical controls). Allocated scores were compared, and consensus was reached by discussion. Data abstraction was per- formed by one of the authors (BW) and independently cross-checked by the other two.

\section{Qualitative, Quantitative, and Subgroup Analyses}

For colonization, we calculated relative risk (RR) with $95 \%$ confidence interval $\left(\mathrm{CI}_{95}\right) \cdot{ }^{18}$ For BSI, which happens rarely, we calculated Peto odds ratio (OR); this is a more appropriate model when many trials show no events. ${ }^{19}$ We tested for statistical heterogeneity using a chisquare test. A fixed effect model was used throughout because we combined data only when they were clinically homogeneous. ${ }^{20}$

There was a pre hoc agreement that an absolute risk reduction of $2 \%$ or more to prevent BSI (corresponding to a number-needed-to-treat ${ }^{21}$ of 50 or fewer) was a clinically relevant result ${ }^{2}$ and, therefore, would justify the use of antiinfective CVCs.

Sensitivity analyses were performed to test the impact of the duration of catheterization on the efficacy of these anti-infective devices to prevent BSI. For this purpose, we extracted information on the average catheter insertion times from the original articles.

\section{RESULTS \\ Trial Characteristics}

A total of 103 reports were screened. Twenty-seven were considered for inclusion and 4 were subsequently excluded due to the following: one had an inadequate control group ${ }^{22}$; one used historical controls ${ }^{23}$; one had inadequate endpoints ${ }^{24}$; and one ${ }^{25}$ described the same cohort reported in a previous article. ${ }^{26}$ Finally, 23 trials ${ }^{8,9,2646}$ published between 1988 and 1999 including data on 4,660 catheters (2,319 anti-infective and 2,341 control) were analyzed (Table 1). Eleven were conducted in an intensive care unit setting, four were conducted among oncologic patients, two were conducted among surgical patients, two were conducted among patients receiving total parenteral nutrition, and four were conducted among other patient populations. The average number of catheters per trial was 194 (range, 20 to 680). The dropout rate of catheters varied between $0 \%$ and $49 \%$. Average observation periods ranged from 2 to 147 days. The median Oxford score was 2 (range, 1 to 5). In 18 trials, no attempt was made at blinding.

Anti-infective catheters were treated with chlorhexidine-silver sulfadiazine coating (12 trials, 1,456 catheters); silver-impregnated collagen cuffing (5 trials, 328 catheters); antibiotic coating with teicoplanin, minocycline plus rifampin, vancomycin, or cefazolin (5 trials, 329 catheters); and silver coating ( 2 trials, 206 catheters). One trial compared two devices with an inactive control group ${ }^{41}$; all other trials compared one device with a control.

\section{Qualitative and Quantitative Analyses}

Catheter Colonization. Catheter colonization was reported in 22 trials ( $3,902 \mathrm{CVCs}$ ) (Table 1$)$. There was a large variability in event rates with both anti-infective and control catheters: $1.7 \%$ to $62 \%$ (average, $22 \%$ ) for anti-infec- 


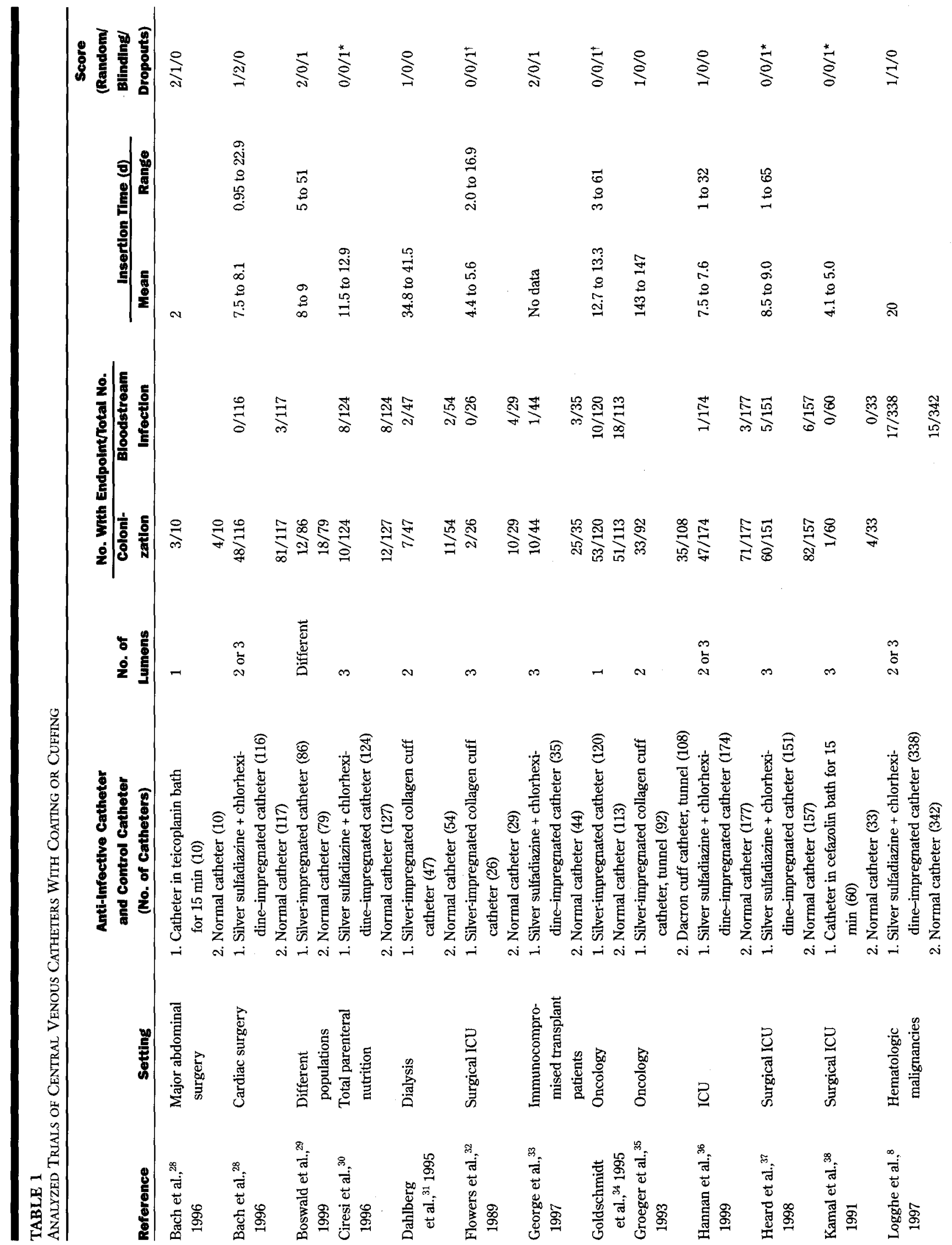




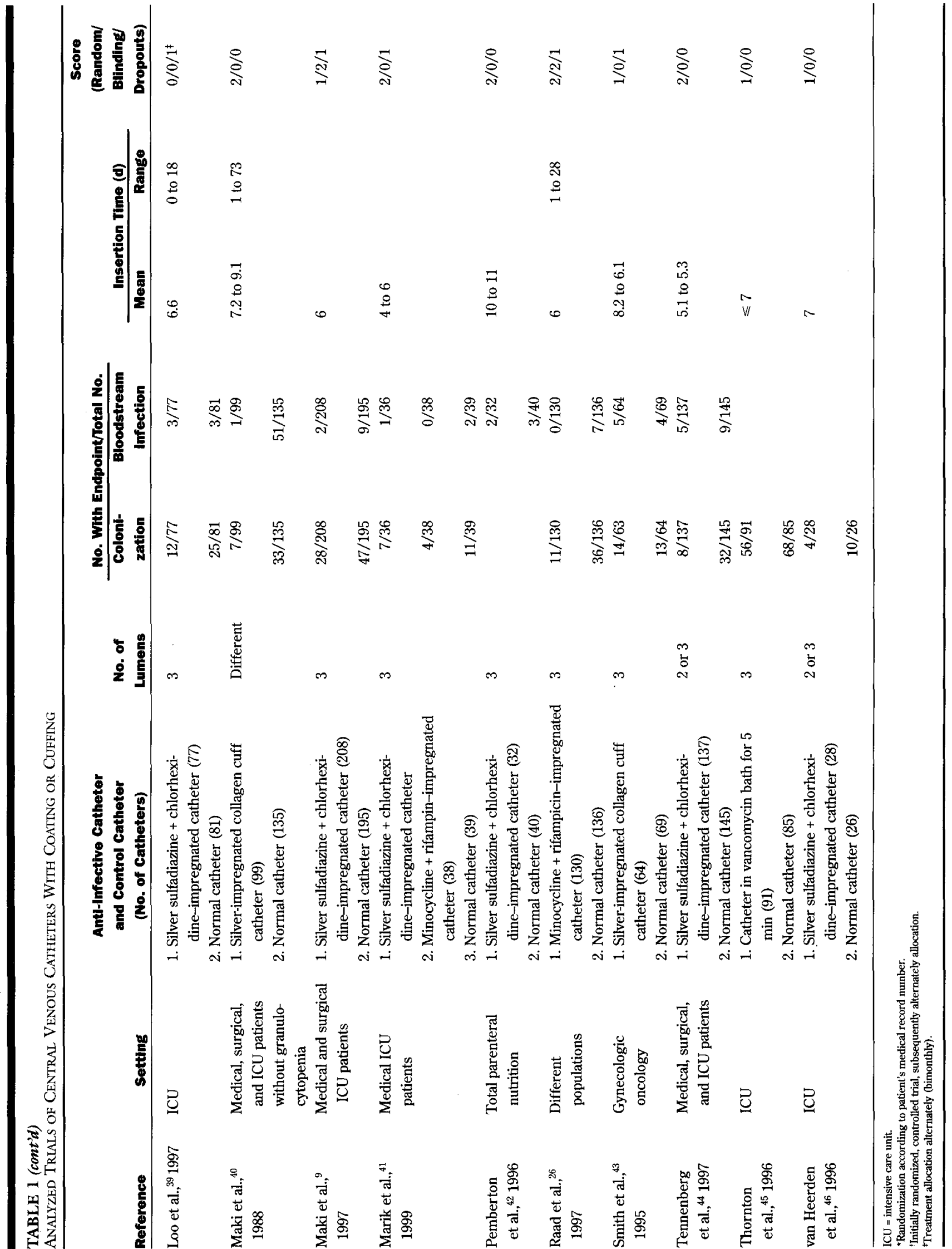




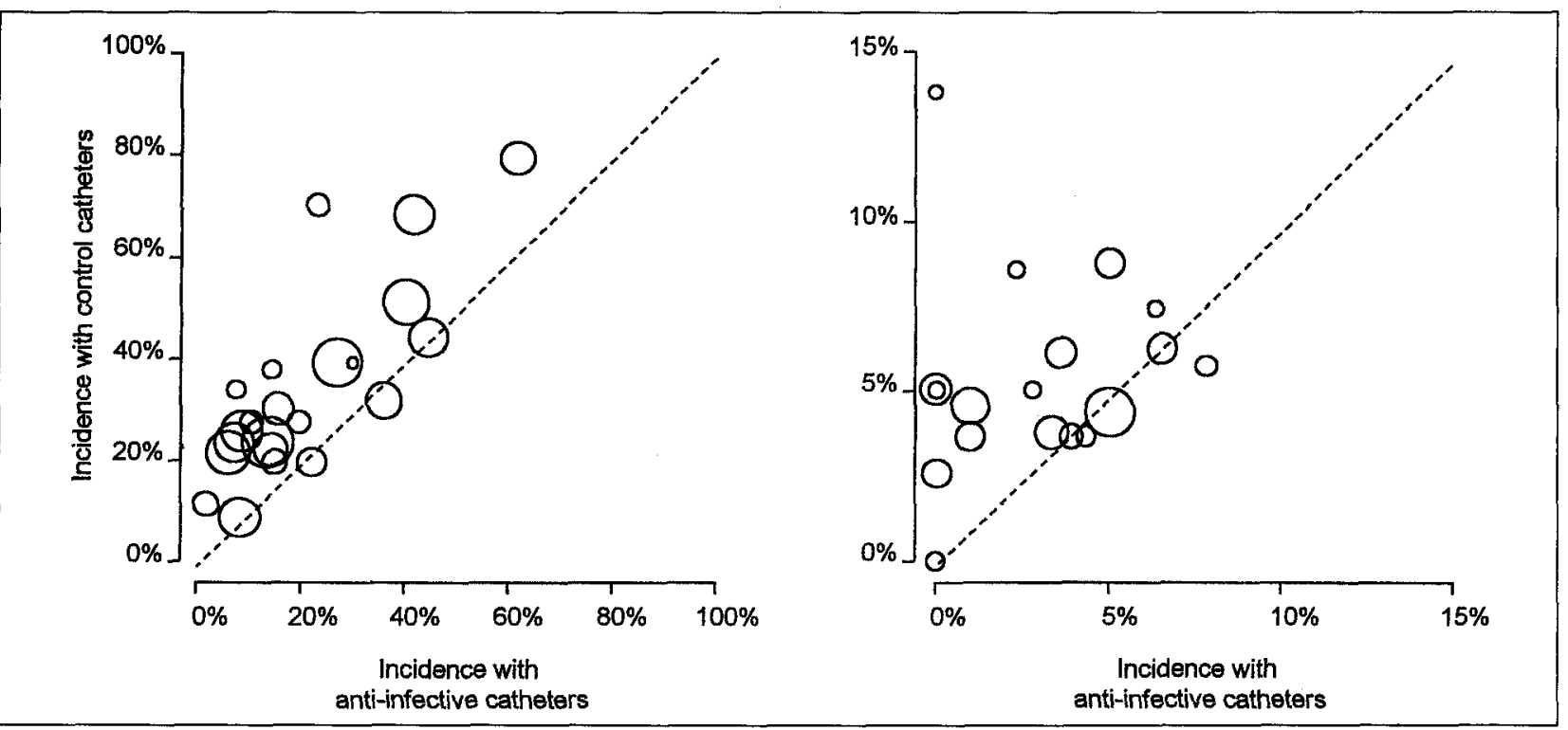

FIGURE 1. Incidence of (left) colonization and (right) bloodstream infection with anti-infective catheters ( $x$ axis) and control catheters ( $y$ axis). Each circle represents one trial. The sizes of the circles are proportional to the sizes of the trials. The dotted lines represent equality.

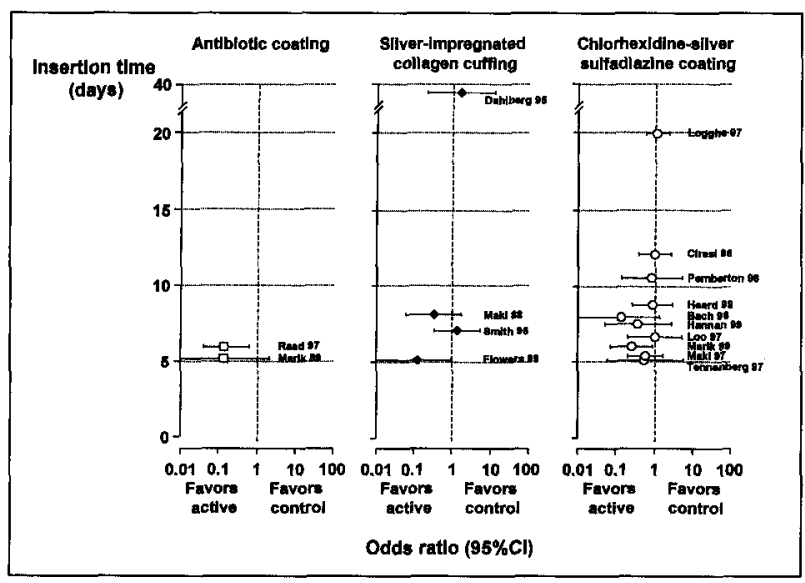

FIGURE 2. Impact of the average insertion times as reported in the original trials on the efficacy of anti-infective catheters to prevent bloodstream infection in the respective study. One study ${ }^{38}$ with no events has been excluded. $95 \% \mathrm{Cl}=95 \%$ confidence interval.

tive catheters and $9.4 \%$ to $80 \%$ (average, $35 \%$ ) for control catheters (Fig. 1, left). The scatter suggested improvement with anti-infective catheters. The data were heterogeneous $(P<.001)$. When data from all trials were combined, the difference in favor of anti-infective catheters was statistically significant ( $\mathrm{RR}, 0.61 ; \mathrm{CI}_{95}, 0.51$ to 0.72 ).

BSI. BSI was reported in 18 trials $(4,045 \mathrm{CVCs})$ (Table 1). There was a large variability in event rates with both anti-infective and control catheters: $0 \%$ to $7.8 \%$ (average, $2.9 \%$ ) for anti-infective catheters and $0 \%$ to $13.8 \%$ (average, 4.7\%) for control catheters (Fig. 1, right). In one trial, $4(13.8 \%)$ of 29 patients receiving an inactive control catheter had a BSI, whereas none of 26 receiving a silverimpregnated collagen cuff catheter did. ${ }^{32}$ The scatter suggested improvement with anti-infective catheters. The data were homogeneous $(P=.329)$. When data from all trials were combined, the difference in favor of anti-infective catheters was statistically significant $\left(\mathrm{OR}, 0.63 ; \mathrm{CI}_{95}, 0.45\right.$ to $0.87)$.

Impact of Insertion Time on Anti-Infective Efficacy. Of all 18 trials that reported on BSI, one $\mathrm{e}^{33}$ did not report on the average insertion time, one ${ }^{34}$ tested silver coating (ie, a technique that was not tested in any other trial reporting on $\mathrm{BSI}$ ), and in on $\mathrm{e}^{38}$ there was no BSI (ie, 0 events). Thus, sensitivity analyses to quantify the impact of insertion time on anti-infective efficacy could be performed with data from 15 trials (16 comparisons) that tested antibiotic coating, silver-impregnated collagen cuffing, and chlorhexidine-silver sulfadiazine coating (Fig. 2; Table 2).

In both relevant trials on antibiotic coating ( 343 CVCs), the average insertion time was 6 days. ${ }^{26,41}$ The combined risk of BSI decreased from $5.1 \%$ with control to $0 \%$ with anti-infective catheters ( $\mathrm{OR}, 0.14 ; \mathrm{CI}_{95}, 0.04$ to 0.51 ). There were no trials with longer average insertion times.

In three of four trials on silver-impregnated collagen cuffs $(422 \mathrm{CVCs})$, the average insertion time ranged from 5 to 8.2 days (median, 7 days). ${ }^{32,40,43}$ The combined risk of BSI was $5.6 \%$ with control and $3.2 \%$ with anti-infective catheters (OR, $0.54 ; \mathrm{CI}_{95}, 0.21$ to 1.36 ). In the fourth trial on silver-impregnated collagen cuffs $(101 \mathrm{CVCs})$, the average insertion time was 38 days. ${ }^{31}$ The risk of BSI was $3.7 \%$ with control and $4.3 \%$ with anti-infective catheters (OR, 1.15; $\mathrm{CI}_{95}, 0.16$ to 8.49 ). One trial reported an average insertion time of 8.2 days. ${ }^{40}$ When data from this trial were pooled with the data from the trial with the longest average insertion time, ${ }^{31}$ long-term efficacy was still not statistically significant (incidence of BSI, $3.7 \%$ with control vs $2.1 \%$ with anti-infective catheters [OR, $0.56 ; \mathrm{CI}_{95}, 0.16$ to 1.98$]$ ).

In five of 10 trials on chlorhexidine-silver sulfadiazine coating $(1,269 \mathrm{CVCs})$, the average insertion time 
ranged from 5.2 to 7.5 days (median, 6 days). ${ }^{936,39,41,44}$ The combined risk of BSI decreased from $4.1 \%$ with control to $1.9 \%$ with anti-infective catheters $\left(\mathrm{OR}, 0.48 ; \mathrm{CI}_{95}, 0.25\right.$ to 0.91 ). In the five other trials on chlorhexidine-silver sulfadiazine coating $(1,544 \mathrm{CVCs})$, the average insertion time ranged from 7.8 to 20 days (median, 12 days). $8,28,30,37,42$ The combined risk of BSI was $4.5 \%$ with control and $4.2 \%$ with anti-infective catheters $\left(\mathrm{OR}, 0.94 ; \mathrm{CI}_{95}, 0.58\right.$ to 1.54$)$.

\section{Adverse Events}

Twelve trials reported the presence or absence of adverse events. $926,27,31-34,38,40,42,43,45$ There was some evidence that cuffed catheters were more difficult to insert than control catheters, ${ }^{31}$ and that they extruded more often. ${ }^{32,40,43}$ No systemic allergic reaction or local hypersensitivity was reported in any trial.

\section{Conclusions of the Original Authors}

In 12 studies (52\%), the original authors concluded from their data that anti-infective catheters were useful. $9,26,29,32,34,36,38-41,43,45$ In ten, they questioned the usefulness of such devices. The authors of one trial did not draw any conclusions. ${ }^{46}$

\section{DISCUSSION}

One of the proposed strategies to prevent CVC-related infection is the antiseptic or antimicrobial coating or cuffing of catheters ${ }^{47} \mathrm{BSI}$ is the most relevant endpoint in this context. Two factors can be identified that have a major impact on the prevention of BSI with these catheters: insertion time and type of anti-infective method. In previous meta-analyses on the antimicrobial efficacy of catheters treated with chlorhexidine-silver sulfadiazine, and from data on short-term trials only (2 to 10 days), there was a statistically significant decrease in BSI compared with control catheters (OR, 0.56; $\mathrm{CI}_{95}, 0.37$ to 0.84$){ }_{.}^{11,12,47}$ The authors concluded that these devices were effective in decreasing BSI. In our analysis, the result was similar for the subgroup of catheters treated with chlorhexidine-silver sulfadiazine and having short-term insertion $\left(\mathrm{OR}, 0.48 ; \mathrm{CI}_{95}, 0.25\right.$ to $0.91)$. However, one additional important conclusion from this systematic review is that first-generation chlorhexidine-silver sulfadiazine catheters are not efficacious when the average insertion time is longer than 8 days.

Microorganisms in contact with plastic surfaces produce a protective, multi-layer biofilm within which they can survive. ${ }^{48}$ It is tempting to speculate that such a biofilm could suppress the anti-infective action of the treated catheters. Indeed, with average insertion times of approximately 1 week, 1 in approximately 50 catheters treated with chlorhexidine-silver sulfadiazine will not lead to a BSI, which would have been the case with inactive devices (absolute risk reduction, 2.2\%) (Table 2). When average insertion times were twice as long (approximately 12 days), this number was 1 in approximately 300 (absolute risk reduction, $0.3 \%$ ). These results are in agreement with both in vitro and in vivo studies. ${ }^{13,28}$ In vitro, a steep exponential decrease in antimicrobial activity was shown during the

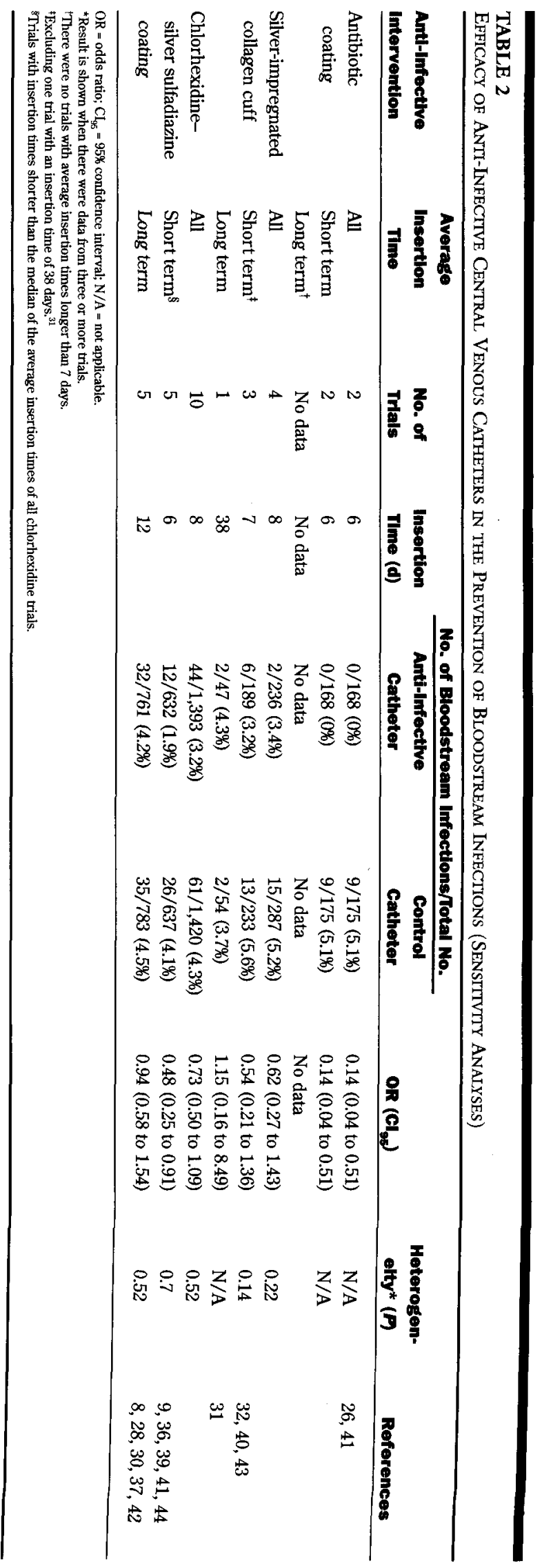


first week of insertion for devices treated with chlorhexidine-silver sulfadiazine. ${ }^{28}$ In vivo, the antimicrobial activity of CVCs treated with chlorhexidine-silver sulfadiazine was shown to be time dependent. ${ }^{13}$

With antibiotic coating, there was not one single case of BSI. The absolute risk reduction compared with inactive control catheters was $5.1 \%$, suggesting that approximately 20 patients need to receive an antibioticcoated CVC for one patient not to develop a BSI who would have done so had they received inactive catheters. Thus, for short insertion times (ie, no longer than approximately 1 week), there was some evidence from indirect comparisons that antibiotic coating was more efficacious than chlorhexidine-silver sulfadiazine impregnation. These data are consistent with the results of a recently published large, randomized trial ${ }^{13}$ in which CVC-related BSI was less likely in catheters coated with minocycline-rifampin (incidence, $0.3 \%$ ) compared with catheters treated with chlorhexidine-silver sulfadiazine (incidence, $3.4 \%$ ) with a median insertion time of 6 to 7 days. For both devices, the degree of short-term antimicrobial efficacy falls into our pre hoc definition of worthwhile prevention (ie, absolute risk reduction of $\geqslant 2 \%$ corresponding to a number-neededto-treat of $\leqslant 50$ ). However, data from the randomized trial $^{13}$ and from our meta-analysis suggest that twice as many catheters treated with chlorhexidine-silver sulfadiazine, compared with antibiotic-coated catheters, need to be inserted to prevent one BSI. We do not know whether the difference in efficacy between these catheter types is due to the anti-infective substances per se, different methods of coating, or both. Impregnation with chlorhexidine-silver sulfadiazine covers only the external surface of catheters. Some antibiotic coatings cover both the external and the internal surfaces of catheters. This may be associated with enhanced efficacy, as previously suggested, ${ }^{13}$ but needs to be formally tested.

No long-term data on antibiotic-treated CVCs were available. In one study, a complete loss of activity for teicoplanin-coated catheters within 36 hours was reported. ${ }^{27}$ Catheters treated with minocycline and rifampin may have antimicrobial activity extending up to 2 and possibly up to 4 to 6 weeks. ${ }^{25}$ This inconsistency may be interpreted as evidence of specific antibiotic activities. Further randomized trials are needed to establish the effect of different antibiotic coating methods on the long-term anti-infective efficacy of CVCs.

Silver-impregnated collagen cuffing did not prevent BSI, although one small trial with a short insertion time (average, 5 days) reported a borderline significant result in favor of the anti-infective catheters. ${ }^{32}$ In that trial, almost $14 \%$ of patients who received a control catheter had a BSI, a rate that was much higher than that in any other trial (Fig. 1 , right). Because we have to assume that there is a relationship between the event rate in control catheters and the true underlying risk, it may be speculated that that trial studied patients at a particular high risk of infection. This was not the case (Table 1). However, small trials have been shown in other settings to overestimate the effect of an intervention. ${ }^{49}$ Because that trial was of limited size, it had no important weight in the combined analysis.

We had to rely on average insertion times as reported in the original studies to estimate the potential impact of insertion time on the efficacy of anti-infective CVCs. In some, the range of catheter duration for individual patients was wide. This is a limitation of our study. However, in the absence of valid randomized trials comparing different insertion times, these analyses remain the best we have. It would be helpful to confirm our results using individual patient data.

Assuming that some anti-infective catheters prevent BSI if they are kept in situ for less than 1 week, we have to ask whether it is worthwhile to exchange these catheters regularly in chronically catheterized patients. There are three issues here: device, risk, and cost. Within the limitations of our analysis, there was evidence for time dependency with catheters coated with chlorhexidine-silver sulfadiazine. Thus, if those devices are to be used, there is an argument to replace them regularly, although it is not known whether one replacement per week is optimal. Systematic replacement of conventional CVCs through guidewire exchange every 3 days did not seem to reduce the risk of infection in a randomized trial..$^{50}$ There was no evidence for time dependency of antibiotic-coated devices because no trials reporting on longer average insertion times could be retrieved. If the antimicrobial efficacy of antibiotic-coated catheters should indeed extend to 2 to 4 weeks, ${ }^{13,25}$ an exchange of them after 7 days might not be necessary. However, this needs to be confirmed in randomized, controlled trials.

Risk includes mechanical complications during insertion, ${ }^{1}$ allergic reactions, and resistance acquisition. Insertion of cuffed catheters may be more difficult, and extrusion may occur. ${ }^{32,40,43}$ Severe allergic reactions related to catheters coated with chlorhexidine-silver sulfadiazine have been described ${ }^{51,52}$; however, no systemic allergic reactions were reported in the analyzed trials, most likely due to their low incidence. It is likely that the issue of increased risk of resistance concerns mostly antibioticcoated catheters. Furthermore, whether long-term exposure of bacteria to disinfectants is associated with increasing resistance or possible selective pressure to become antibiotic resistant remains to be determined. ${ }^{53}$

Anti-infective catheters cost approximately twice as much as conventional catheters. To justify a change in clinical practice, the increased cost incurred by a more frequent use of a more expensive device must be balanced against the savings obtained by preventing BSIs. Catheters coated with chlorhexidine-silver sulfadiazine appeared to be cost-effective for short-term insertion (2 to 10 days). ${ }^{12}$ Antibiotic-coated catheters were recommended for infection control in critically ill patients. ${ }^{14}$ However, the economic impact of these CVCs should be evaluated to enable policy decisions to be made regarding their implementation in clinical practice.

When inserted for no longer than 1 week, 1 in approximately 20 antibiotic-coated catheters and 1 in 
approximately 50 catheters coated with chlorhexidine-silver sulfadiazine will not lead to a BSI, which would have occurred had they not been coated. As recently emphasized, this novel technology should be viewed as an adjunct to, rather than a substitute for, good infection control practices. ${ }^{54}$ Stringent application of guidelines to prevent catheter infection has been recognized as highly efficacious in critically ill patients. ${ }^{55}$ Anti-infective catheters are important in reducing the incidence of CVC-related infection, but further research is required to define the most efficacious method and the optimal time for device replacement and to identify subgroups of patients who might benefit most from this protective measure. Cost-effectiveness analyses of such interventions are also required.

\section{REFERENCES}

1. Ruesch $S$, Walder $B$, Tramèr MR. Complications of central venous catheters: internal jugular versus subclavian access-a systematic review. Crit Care Med 2002;30:454-460.

2. Maki DG, Mermel LA. Infections due to infusion therapy. In: Bennett JV Brachman PS, eds. Hospital Infections, 4th ed. Philadelphia: LippincottRaven; 1998:689-724.

3. Arnow PM, Quimosing EM, Beach M. Consequences of intravascular catheter sepsis. Clin Infect Dis 1993;16:778-784.

4. Jarvis W, Edwards J, Culver D, et al. Nosocomial infection rates in adults and pediatric intensive care units in the United States: the National Nosocomial Infections Surveillance System. Am J Med 1991;91:185S 191S.

5. Pittet D. Nosocomial bloodstream infections. In: Wenzel RP, ed. Prevention and Control of Nosocomial Infections, 3rd ed. Baltimore: Williams and Wilkins; 1997:711-769.

6. Richet H, Hubert B, Nitemberg G, et al. Prospective multicenter study of vascular-catheter-related complications and risk factors for positive central-catheter cultures in intensive care unit patients. J Clin Microbiol 1990;28:2520-2525.

7. Pittet D, Tarara D, Wenzel RP. Nosocomial bloodstream infection in critically ill patients: excess length of stay, extra costs, and attributable mortality. JAMA 1994;271:1598-1601.

8. Logghe C, Van Ossel C, D'Hoore W, Ezzedine H, Wauters G, Haxhe JJ Evaluation of chlorhexidine and silver-sulfadiazine impregnated central venous catheters for the prevention of bloodstream infection in leukaemic patients: a randomized controlled trial. J Hosp Infect 1997; 37:145-156.

9. Maki DG, Stolz SM, Wheeler S, Mermel LA. Prevention of central venous catheter-related bloodstream infection by use of an antisepticimpregnated catheter: a randomized, controlled trial. Ann Intern Med 1997;127:257-266

10. Pearson ML. Guideline for prevention of intravascular device-related infections: Part I. Intravascular device-related infections: an overview. The Hospital Infection Control Practices Advisory Committee. Am J Infect Control 1996;24:262-277.

11. Veenstra DI, Saint S, Saha S, Lumley T, Sullivan SD. Efficacy of antiseptic-impregnated central venous catheters in preventing catheter-related bloodstream infection. JAMA 1999;281:261-267.

12. Veenstra DL, Saint S, Sullivan SD. Cost-effectiveness of antisepticimpregnated central venous catheters for the prevention of catheterrelated bloodstream infection. JAMA 1999;282:554-560.

13. Darouiche RO, Raad I, Heard SO, et al. A comparison of two antimicrobial-impregnated central venous catheters. $N$ Engl J Med 1999;340:1-8.

14. Wenzel RP, Edmond MB. The evolving technology of venous access. $N$ Engl J Med 1999;340:48-50.

15. Haxhe JJ, D'Hoore W. A meta-analysis dealing with the effectiveness of chlorhexidine and silver-sufhadiazine impregnated central venous catheters. J Hosp Infect 1998;40:166-168.

16. Maki DG, Weise CE, Sarafin HW. A semiquantitative culture method for identifying intravenous-catheter-related infection. N Engl J Med 1977; 296:1305-1309.

17. Jadad AR, Moore RA, Carroll D, et al. Assessing the quality of reports of randomized clinical trials: is blinding necessary? Control Clin Trials 1996;17:1-12.

18. Morris JA, Gardner MJ. Statistics with confidence: confidence intervals and statistical guideline. In: Gardner MJ, Altman DG, eds. Calculating Confidence Intervals for Relative Risk, Odds Ratios, and Standardised Ratios and Rates. London: British Medical Journal; 1995:50-63.
19. Yusuf $S$, Peto $R$, Lewis J. Beta blockade during and after myocardial infarction: an overview of the randomized trials. Progress in Cardiovascular Research 1985;27:335-371.

20. Gavaghan DJ, Moore RA, McQuay HJ. An evaluation of homogeneity tests in meta-analyses in pain using simulations of individual patient data. Pain 2000;85:415-424.

21. Laupacis A, Sackett DL, Roberts RS. An assessment of clinically useful measures of the consequences of treatment. $N$ Engl J Med 1988;318: 1728-1733.

22. Babycos CR, Barrocas A, Webb WR A prospective randomized trial comparing the silver-impregnated collagen cuff with the bedside tunneled subclavian catheter. JPEN J Parenter Enteral Nutr 1993;17:61-63.

23. Hasaniya NW, Angelis M, Brown MR, Yu M. Efficacy of subcutaneous silver-impregnated cuffs in preventing central venous catheter infections. Chest 1996;109:1030-1032.

24. Bambauer R, Schiel R, Mestres P, Klinkmann J, Sioshansi P. Scanning electron microscopic investigation of catheters for blood access. Blood Purif 1996;14:249-256.

25. Raad II, Darouiche RO, Hachem R, et al. Antimicrobial durability and rare ultrastructural colonization of indwelling central catheters coated with minocycline and rifampin. Crit Care Med 1998;26:219-224.

26. Raad I, Darouiche R, Dupuis J, et al. Central venous catheters coated with minocycline and rifampin for the prevention of catheter-related colonization and bloodstream infections: a randomized, double-blind trial. The Texas Medical Center Catheter Study Group. Ann Intern Med 1997; 127:267-274.

27. Bach A, Darby D, Bottiger B, Bohrer H, Motsch J, Martin E. Retention of the antibiotic teicoplanin 'on a hydromer-coated central venous catheter to prevent bacterial colonization in postoperative surgical patients. Intensive Care Med 1996;22:1066-1069.

28. Bach A, Schmidt H, Bottiger B, et al. Retention of antibacterial activity and bacterial colonization of antiseptic-bonded central venous catheters. J Antimicrob Chemother 1996;37:315-322.

29. Boswald M, Lugauer S, Regenfus A, et al. Reduced rates of catheterassociated infection by use of a new silver-impregnated central venous catheter. Infection 1999;27:\$56-S60.

30. Ciresi DL, Albrecht RM, Volkers PA, Scholten DJ. Failure of antiseptic bonding to prevent central venous catheter-related infection and sepsis. Am Surg 1996;62:641-646.

31. Dahlberg PJ, Agger WA, Singer JR, et al. Subclavian hemodialysis catheter infections: a prospective, randomized trial of an attachable silver-impregnated cuff for prevention of catheter-related infections. Infect Control Hosp Epidemiol 1995;16:506-511.

32. Flowers RH 3rd, Schwenzer KJ, Kopel RF, Fisch MJ, Tucker SI, Farr BM. Efficacy of an attachable subcutaneous cuff for the prevention of intravascular catheter-related infection: a randomized, controlled trial. JAMA 1989;261:878-883.

33. George SJ, Vuddamalay P, Boscoe MJ. Antiseptic-impregnated central venous catheters reduce the incidence of bacterial colonization and associated infection in immunocompromised transplant patients. Eur J Anaesthesiol 1997:14:428-431.

34. Goldschmidt H, Hahn U, Salwender HJ, et al. Prevention of catheterrelated infections by silver coated central venous catheters in oncological patients. Zentralbl Bakteriol 1995;283:215-223.

35. Groeger JS, Lucas AB, Coit D, et al. A prospective, randomized evaluation of the effect of silver impregnated subcutaneous cuffs for preventing tunneled chronic venous access catheter infections in cancer patients. Ann Surg 1993;218:206-210.

36. Hannan M, Juste RN, Umasanker S, et al. Antiseptic-bonded central venous catheters and bacterial colonisation. Anaesthesia 1999;54:868 872.

37. Heard SO, Wagle M, Vijayakumar E, et al. Influence of triple-lumen central venous catheters coated with chlorhexidine and silver sulfadiazine on the incidence of catheter-related bacteremia. Arch Intern Med 1998;158:81-8?

38. Kamal GD, Pfaller MA, Rempe LE, Jebson PJ. Reduced intravascular catheter infection by antibiotic bonding: a prospective, randomized, controlled trial. JAMA 1991;265:2364-2368.

39. Loo S, VanHerden PV, Gollege CL, et al. Infection in central lines: antiseptic-impregnated vs standard non-impregnated catheters. Anaesth Intensive Care 1997;25:637-639.

40. Maki DG, Cobb L, Garman JK, Shapiro JM, Ringer M, Helgerson RB. An attachable silver-impregnated cuff for prevention of infection with central venous catheters: a prospective randomized multicenter trial. $\mathrm{Am} \mathrm{J}$ Med 1988;85:307-314.

41. Marik PE, Abraham G, Careau P, Varon J, Fromm RE Jr. The ex vivo antimicrobial activity and colonization rate of two antimicrobial-bonded central venous catheters. Crit Care Med 1999;27:1128-1131.

42. Pemberton LB, Ross V, Cuddy P, Kremer H, Fessler T, McGurk E. No difference in catheter sepsis between standard and antiseptic central 
venous catheters: a prospective randomized trial. Arch Surg $1996 ; 131: 986-989$.

43. Smith HO, DeVictoria CL, Garfinkel D, et al. A prospective randomized comparison of an attached silver-impregnated cuff to prevent central venous catheter-associated infection. Gynecol Oncol 1995;58:92-100.

44. Tennenberg S, Lieser M, McCurdy B, et al. A prospective randomized trial of an antibiotic- and antiseptic-coated central venous catheter in the prevention of catheter-related infections. Arch Surg 1997;132:1348-1351.

45. Thornton J, Todd NJ, Webster NR. Central venous line sepsis in the intensive care unit: a study comparing antibiotic coated catheters with plain catheters. Anaesthesia 1996;51:1018-1020.

46. van Heerden PV, Webb SA, Fong S, et al. Central venous catheters revisited: infection rates and an assessment of the new Fibrin Analysing System Brush. Anaesth Intensive Care 1996;24:330-333.

47. Raad I. Intravascular-catheter-related infections. Lancet 1998;351:893898.

48. Lew DP, Pittet D, Waldvogel FA. Infections that complicate the insertion of prosthetic devices. In: Mayhall CG, ed. Hospital Epidemiology and Infection Control, 2nd ed. Baltimore: Williams and Wilkins; 1996:731748.

49. Moore RA, Tramèr MR, Carroll D, Wiffen PJ, McQuay HJ. Quantitative systematic review of topically applied non-steroidal anti-inflammatory drugs. $B M J$ 1998;316:333-338.

50. Cobb DK, High KP, Sawyer RG, et al. A controlled trial of scheduled replacement of central venous and pulmonary-artery catheters. $N$ Engl J Med 1992;327:1062-1068.

51. Oda T, Hamasaki J, Kanda N, Mikami K Anaphylactic shock induced by an antiseptic-coated central nervous catheter. Anesthesiology 1997;87:1242-1244.

52. Terazawa E, Shimonaka H, Nagase K, Masue T, Dohi S. Severe anaphylactic reaction due to a chlorhexidine-impregnated central venous catheter. Anesthesiology 1998;89:1296-1298.

53. Sivaji Y, Mandal A, Agarwal DS. Disinfectant induced changes in the antibiotic sensitivity and phage typing pattern in Staphylococcus aureus. J Hosp Infect 1986;7:236-243.

54. Pearson ML, Abrutyn E. Reducing the risk for catheter-related infections: a new strategy. Ann Intern Med 1997;127:304-306.

55. Eggimann P, Harbarth S, Constantin MN, Touveneau S, Chevrolet JC Pittet $D$. Impact of a prevention strategy targeted at vascular-access care on incidence of infections acquired in intensive care. Lancet 2000;355:1864-1868. 